\title{
Avaliação da redução da carga microbiana de droga vegetal através do processamento tecnológico: decocção e secagem por aspersão
}

\author{
Tatiane P. de Souza ${ }^{1,2 *}$, Maria I. Zulian Lionzo ${ }^{1}$, Pedro R. Petrovick ${ }^{1}$ \\ ${ }^{1}$ Laboratório de Desenvolvimento Galênico, Faculdade de Farmácia, Universidade Federal do Rio Grande do Sul, \\ Av. Ipiranga 2752, 90610-000, Porto Alegre, RS, Brasil, \\ ${ }^{2}$ Departamento de Farmácia, Universidade Federal do Rio Grande do Norte, Av. Gustavo Cordeiro de Farias s/n, \\ Petrópolis, 59010-180, Natal, RN, Brasil
}

\begin{abstract}
RESUMO: As plantas medicinais podem representar importantes alternativas terapêuticas. No entanto, para elaboração de um fitoterápico a contaminação microbiana constitui um problema a ser vencido. O processamento tecnológico da matéria-prima envolve etapas, geralmente, desfavoráveis à sobrevivência de microrganismos, sendo sua eliminação dependente da carga microbiana inicial e das condições de trabalho utilizadas. Assim, este estudo teve como objetivo verificar a contaminação microbiana presente nas partes aéreas de Phyllanthus niruri e produtos derivados, solução extrativa (SE) e produto seco por aspersão (PSA), a fim de avaliar a redução da contaminação após a decocção e a secagem por aspersão. A determinação dos microrganismos viáveis nos produtos foi realizada através do método de contagem em placas, e a quantificação de coliformes totais pela técnica do número mais provável. Os resultados demonstraram que carga microbiana dos produtos analisados encontrava-se abaixo do limite máximo permitido. Em relação à droga vegetal, a solução extrativa apresentou carga microbiana consideravelmente menor, sugerindo que a decocção tenha sido responsável pela redução de $98,3 \%$ da contaminação inicial. Por outro lado, a carga microbiana do PSA foi semelhante a da SE, indicando que os microrganismos não são afetados pela operação de secagem por aspersão.
\end{abstract}

Unitermos: Phyllanthus niruri, contaminação microbiana, solução extrativa, produto seco por aspersão.

\begin{abstract}
Evaluation of microbial contamination reduction on plants through technological process of decoction and spray dry". Medicinal plants can be important therapeutic alternative, however microbiological contamination of plants represent a problem to be solved before the production. The technologic process of raw material has many stages, generally, adverse to microbial growth, but its complete elimination depends on the initial and work condition utilized. The aim of this work was to verify the microbial contamination of Phyllanthus niruri aerial parts and derivatives products, such as extractive solution (SE) and spray dried extract (PSA) with the purpose of evaluating the decrease of the contamination after the decoction and the spray dry. The microbiological analysis of the products was performed by total plate count and MPN coliform. The results showed that the contamination of the products was below the limit maxim. The contamination in the SE was significantly lower than in the plant and this fact suggest that the decoction was responsible for a reduction of $98,3 \%$ of the initial contamination. On the other hand, the PSA contamination was similar the SE thus suggesting that the spray dry did not affect the microorganisms present in the initial product.
\end{abstract}

Keywords: Phyllanthus niruri, microbial contamination, extractive solution, spray dried extract.

\section{INTRODUÇÃO}

A utilização de plantas medicinais está em contínua expansão em nível mundial. A crescente busca por agentes terapêuticos derivados de espécies vegetais justifica-se pelo surgimento de doenças, ainda sem tratamento apropriado, e pelo crescimento do conhecimento científico, a respeito dos fitoterápicos, como importantes alternativas terapêuticas (Schenkel et al., 1985, Gossman et al., 1986; Capasso et al., 2000, Guarrera, 2005). No entanto, para o desenvolvimento de um medicamento a partir de uma droga vegetal algumas barreiras necessitam serem vencidas, dentre elas a questão da contaminação microbiana da matéria-prima.

As preparações obtidas a partir de drogas vegetais, geralmente, são oriundas de plantas coletadas, secas e empacotadas sem muitos cuidados de higiene e ou controle sanitário, além disso, as características biológicas e o elevado teor de umidade podem favorecer a contaminação e o crescimento microbiano (List; Schmidt, 1989; Martins et al., 2001). Nesse sentido, a literatura registra que a qualidade microbiológica de matériasprimas vegetais e produtos derivados, geralmente apresenta índices de contaminação microbiana acima dos 
níveis internacionalmente aceitos para medicamentos (Fischer et al., 1996; Limyatti; Juniar, 1998; Araújo; Ohara, 2000).

A produção de um fitoterápico geralmente envolve etapas em que a droga vegetal é submetida a condições desfavoráveis à sobrevivência de microrganismos, tais como extração com solventes orgânicos e ou a elevadas temperaturas. $O$ processo de decocção, por exemplo, em que a droga é colocada em contato com água em ebulição, pode gerar produtos com reduzida carga microbiana, no entanto, dependendo do grau de contaminação na matéria-prima inicial, esse processo pode não ser efetivo (Araújo; Ohara, 2000; Martins et al., 2001). Por outro lado, na operação de secagem por aspersão, método muito utilizado em indústrias farmacêuticas, apesar da elevada temperatura de secagem $\left(100{ }^{\circ} \mathrm{C}\right.$ a $\left.200{ }^{\circ} \mathrm{C}\right)$, o tempo de contato entre o material a ser seco e o ar quente é extremamente rápido, menor que 1 minuto, não servindo, teoricamente, para eliminar os microrganismos viáveis (Broadhead et al., 1992; Remili et al., 1994).

Sendo assim, este trabalho tem por objetivo avaliar a influência das etapas de processamento tecnológico, decocção e secagem por aspersão, na redução da carga microbiana presente numa droga vegetal.

\section{MATERIAL E MÉTODOS}

\section{Tratamento do material}

Droga vegetal: A droga vegetal em estudo foi constituída de partes aéreas de Phyllanthus niruri adquirida na Indústria (Quimer Ltda - São Paulo). O material vegetal recebido apresentava umidade superior a $12 \%$, sendo então, submetido à secagem em estufa de ar circulante, separadamente folhas e caules, a temperatura de $45^{\circ} \mathrm{C} \pm 2{ }^{\circ} \mathrm{C}$, por um período de 7 dias. Após secagem, cada parte do vegetal foi cominuida em moinhos de faca, com abertura de saída de $1 \mathrm{~mm}$, sendo posteriormente misturados para constituir a matéria-prima vegetal.

Solução extrativa (SE): A partir da droga vegetal, foi preparada uma solução extrativa aquosa a $7,5 \%(\mathrm{~m} / \mathrm{V})$ através de decocção, sob refluxo, a $100{ }^{\circ} \mathrm{C}$ por um período de tempo de $15 \mathrm{~min}$ (Soares, 1997).

Produto seco por aspersão (PSA): A solução extrativa foi seca em Mini Spray-drier Büchi, sob as condições de secagem descritas na tabela 1 .

\section{Análise microbiológica}

A análise microbiológica foi realizada através da contagem de microrganismos viáveis totais empregando-se o método de contagem em placas, e pela análise de coliformes totais seguindo a técnica do número mais provável. A SE e o PSA foram analisados imediatamente após preparo. $\mathrm{O}$ ensaio foi repetido em três dias consecutivos.

Contagem de microrganismos viáveis totais (F. Bras. IV, 1988): Cerca de 5g das amostras sólidas e $5 \mathrm{~mL}$ da amostra líquida foram dispersos em $100 \mathrm{~mL}$ de tampão fosfato $\mathrm{pH} 7,2$. Alíquota de $10 \mathrm{~mL}$ da dispersão foi diluída a $100 \mathrm{~mL}$ com água peptonada. Após 1 hora de descanso, $200 \mu \mathrm{L}$ da solução foram esgotados em placa de Petry contendo $20 \mathrm{~mL}$ de meio de cultura ágar caseínasoja, para identificação de bactérias, e incubado durante quatro dias na temperatura de 30 a $35{ }^{\circ} \mathrm{C}$. O mesmo procedimento foi repetido utilizando meio de cultura ágar Sabouraud-Dextrose, para fungos e leveduras, sendo incubado por sete dias na temperatura de 20 a $25^{\circ} \mathrm{C}$. As contagens foram realizadas após incubação.

Número mais provável (NMP) de coliformes totais (F. Bras. IV, 1988): O ensaio foi realizado em três diluições seriadas e três repetições cada (3x3), utilizando como meio de cultura caldo lactosado. Os tubos de ensaio foram incubados em estufa a $30-35^{\circ} \mathrm{C}$, e os resultados foram analisados através da formação de gás após 48 horas de incubação.

Tabela 1. Condições de secagem de mini spray-drier Büchi para obtenção do produto seco por aspersão

\begin{tabular}{l|l}
\hline Parâmetros & Valor \\
\hline Temperatura de entrada & $150{ }^{\circ} \mathrm{C}$ \\
Temperatura de saída & $90{ }^{\circ} \mathrm{C}$ \\
Fluxo de alimentação & $3 \mathrm{~mL} / \mathrm{min}$ \\
Pressão de ar & $2 \mathrm{bar}$ \\
\hline
\end{tabular}

Tabela 2. Contagem de microrganismos viáveis na espécie vegetal de Phyllanthus niruri

GARCIA (2000)

\begin{tabular}{|c|c|c|c|}
\hline Microrganismo & $\begin{array}{l}\text { Dado experimental } \\
\text { (QUIMER) }\end{array}$ & $\begin{array}{l}\text { Planta de crescimento } \\
\text { espontâneo }\end{array}$ & $\begin{array}{l}\text { Planta cultivada } \\
\text { (CPQBA) }\end{array}$ \\
\hline $\begin{array}{l}\text { Bactérias } \\
\text { Fungos }\end{array}$ & $\begin{array}{l}5,7 \times 10^{4} \mathrm{UFC} / \mathrm{g} \\
2,4 \times 10^{4} \mathrm{UFC} / \mathrm{g}\end{array}$ & $\begin{array}{c}5,2 \times 10^{4} \mathrm{UFC} / \mathrm{g} \\
0,45 \times 10^{4} \mathrm{UFC} / \mathrm{g}\end{array}$ & $\begin{array}{l}2,14 \times 10^{4} \mathrm{UFC} / \mathrm{g} \\
0,87 \times 10^{4} \mathrm{UFC} / \mathrm{g}\end{array}$ \\
\hline
\end{tabular}




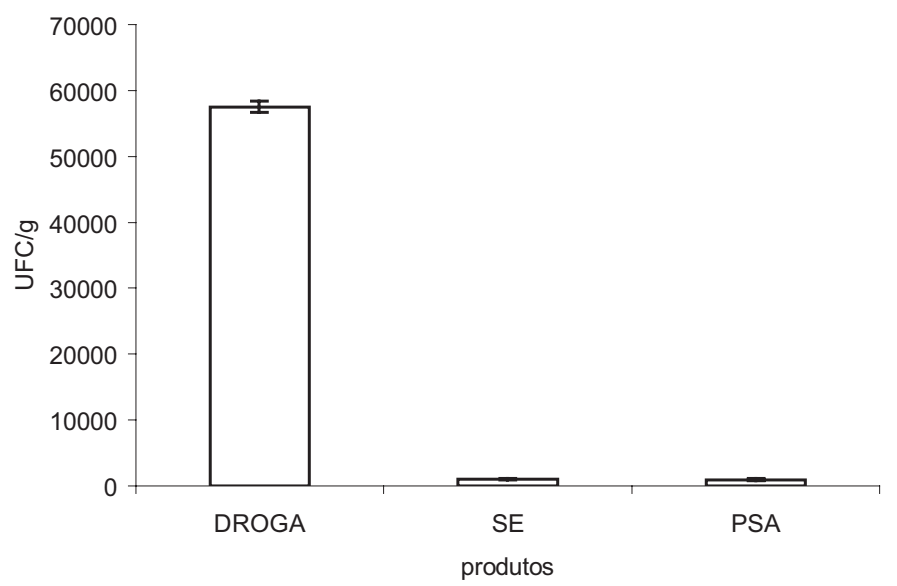

Figura 1. Número de bactérias presentes nos produtos de Phyllanthus niruri

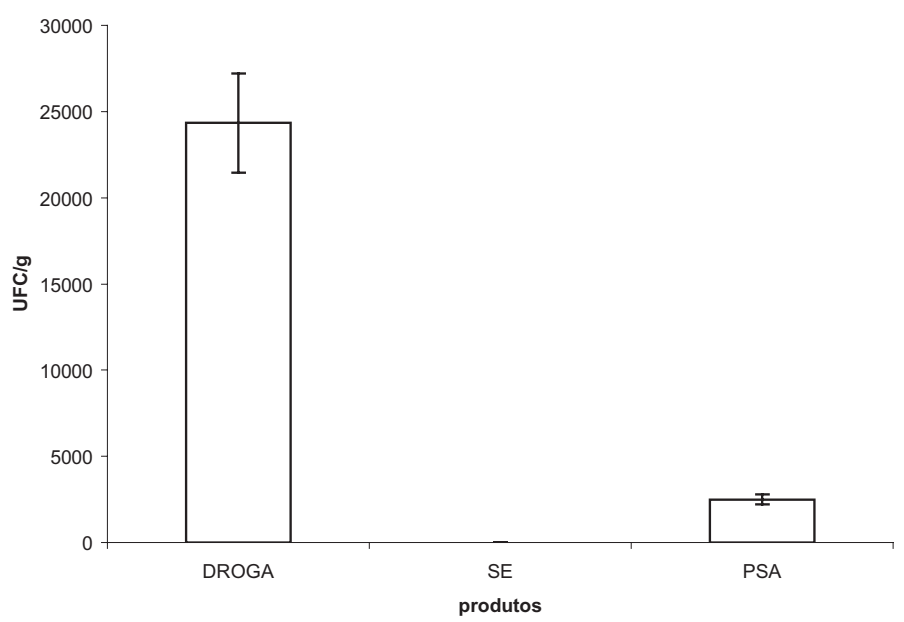

Figura 2. Número de fungos e leveduras presentes nos produtos de Phyllanthus niruri

\section{RESULTADOS E DISCUSSÃO}

A análise microbiológica da droga vegetal foi realizada apenas para determinação da carga microbiana presente na matéria-prima, não sendo identificadas as espécies dos microrganismos. Os resultados da contagem (tabela 2) de bactérias aérobias/mesófilas presentes na droga vegetal indicam contaminação abaixo do limite máximo permitido pela Organização Mundial de Saúde (WHO, 1998) que é de $10^{7} \mathrm{UFC} / \mathrm{g}$ para drogas vegetais. A contagem de fungos e leveduras foi elevada, estando com valor acima do máximo permitido que é de $10^{4}$ $\mathrm{UFC} / \mathrm{g}$. Resultado semelhante foi encontrado por Garcia (2000) que realizou um estudo de avaliação da contagem de microrganismos viáveis em droga vegetal, ou seja, nas partes aéreas de Phyllanthus niruri, de crescimento espontâneo e cultivado pelo Centro Pluridisciplinar de
Pesquisa Químicas, Biológicas e Agrícolas - CPQBA (tabela 2).

A análise microbiológica foi realizada na SE como parâmetro de controle de qualidade e, também, para verificar se o procedimento de decocção era capaz de reduzir a carga microbiana presente na droga vegetal. Vários autores descrevem que o processo de extração, quando realizado a altas temperaturas, pode reduzir significativamente o grau de contaminação do material inicial (De La Rosa et al., 1995; Santos et al., 1995; Araújo; Ohara, 2000; Martins et al., 2001). A carga bacteriana da SE de Phyllanthus niruri, de acordo com a metodologia empregada, foi de $983 \mathrm{UFC} / \mathrm{mL}$ encontrando-se, assim, com um valor bem abaixo do máximo permitido pela Organização Mundial de Saúde (WHO, 1988). Os resultados sugerem que a decocção, provavelmente, foi a razão da redução de $98,3 \%$ no 


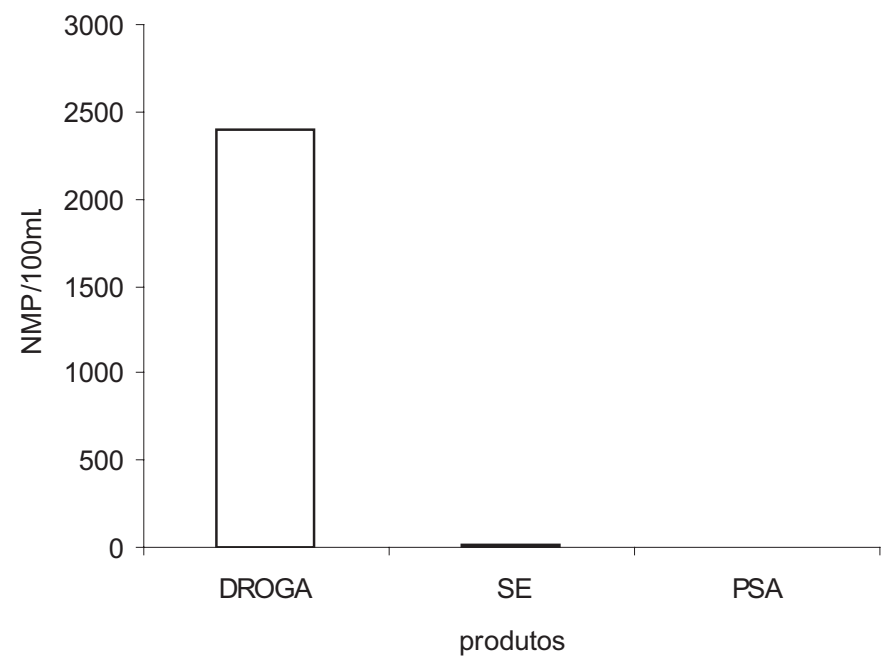

Figura 3. Número mais provável de coliformes totais

grau de contaminação da $\mathrm{SE}$ em relação à droga vegetal. Resultado semelhante foi encontrado por Araújo e Ohara (2000), os quais demonstraram uma diminuição de 99,3\% no nível de contaminação de infusos preparados a partir de drogas vegetais Com relação à presença de fungos e leveduras, a redução foi de $100 \%$, não sendo encontrado na SE nenhuma UFC, tal resultado pode ser devido ao fato da SE ter sido analisada imediatamente após o preparo e, também, pela baixa resistência dos fungos à água em ebulição (Favet, 1992).

A análise microbiológica realizada com PSA demonstrou que a carga de bactérias $\left(9,26 \times 10^{2} \mathrm{UFC} / \mathrm{g}\right)$ e de fungos $\left(2,5 \times 10^{2} \mathrm{UFC} / \mathrm{g}\right)$ encontrava-se abaixo dos limites máximos estabelecidos pela Organização Mundial de Saúde (WHO, 1998), os quais são de $10^{5} \mathrm{UFC} / \mathrm{g}$ e $10^{3} \mathrm{UFC} / \mathrm{g}$ para bactérias e fungos, respectivamente. Quando se compara o nível microbiano do PSA com o da SE pode ser observado que em relação ao número de bactérias parece não haver diferença significativa (figura 1), confirmando o fato de que o processo de secagem por aspersão, em si, não deve alterar os níveis de microrganismos viáveis (Remili et al., 1994), no entanto, a presença de fungos e leveduras no PSA, embora em baixo nível, pode indicar uma contaminação durante a manipulação do produto ou a existência de esporos na $\mathrm{SE}$, uma vez que na mesma o ensaio havia demonstrado ausência de fungos e leveduras (figura 2).

Acarga de coliformes totais foi elevada na droga vegetal (figura 3), no entanto a SE e o PSA apresentaram níveis praticamente desprezíveis, fato provavelmente devido a pouca resistência térmica das bactérias do grupo coliformes (Araújo; Ohara, 2000).

\section{CONCLUSÃO}

Os resultados demonstraram que a droga vegetal adquirida da indústria apresentou grau de contaminação microbiana dentro dos níveis aceitáveis pela Organização Mundial de Saúde para matéria-prima vegetal. O processo de extração ocasionou uma significativa redução no número de microrganismos aeróbios mesófilos viáveis, bem como de fungos e leveduras do material inicial. No entanto, o processo de secagem por aspersão originou um PSA com carga microbiana semelhante da SE, com exceção para o nível de fungos e leveduras o qual estava ausente na SE e presente no PSA. Esse estudo demonstra que o processamento tecnológico da droga vegetal pode contribuir, dependendo das condições utilizadas, para a redução do nível de contaminação microbiana, no entanto maiores estudos devem ser realizados a fim de se determinar a contribuição efetiva de cada etapa do processamento no nível de microrganismos viáveis no produto final.

\section{AGRADECIMENTOS}

Os autores agradecem ao Professor Dr. Carlos Germani e ao Farmacêutico Msc. Marcos Marquard pelo auxílio nas análises microbiológicas. Ao $\mathrm{CNPq}$ e FAPERGS pelo apoio financeiro.

\section{REFERÊNCIAS}

Araújo ALD, Ohara MT 2000. Qualidade microbiológica de drogas vegetais comercializadas em feira de São Paulo e de infusos derivados. Rev Bras Cienc Farmac 36: 129-137.

Broadhead J, Rovan ESK, Rhodes CT 1992. The spray drying of pharmaceuticals. Drug Dev Ind Pharm 18: 11691206.

Capasso R, Izzo AA, Pinto L, Bifulco T, Vitobello C, Mascolo N 2000. Phytotherapy and quality of herbal medicines. Fitoterapia 71: 58-65. 
De La Rosa MC, Medina MR, Vivar C 1995. Microbiological quality of pharmaceutical raw materials. Pharm Acta Helv 70: 227-232.

Farmacopéia Brasileira 1988. 4 ed., São Paulo: Atheneu.

Favet J 1992. Etude de la contamination microbienne d'une vingtaine de drogues végétales. Pharm Acta Helv 67: 250-258.

Fischer DCH, Ohara MT, Saito T 1996. Padrão microbiano em medicamentos não estéreis de uso oral: enquadramento de produtos fitoterápicos. Rev Bras Farmacogn 1: 29-55.

Garcia MC 2000 Determinação da atividade antimicrobiana e caracterização físico-química e biológica de Phyllanthus niruri L. e Phyllanthus tenellus Roxb. (Euphorbiaceae). Dissertação de Mestrado, Curso de Ciência e Tecnologia Farmacêutica, Universidade Federal de Santa Maria.

Gossman G, Faria MR, Santos RI, Vianna RMJ, Simões CMO, Schenkel EP. O controle de qualidade de insumos vegetais e fitoterápicos na Faculdade de Farmácia da UFRGS. Rev Bras Farmacogn 1(Supl 1): 18.

Guarrera MP 2005. Traditional phythoterapy in central Italy. Fitoterapia 76: 1-25.

Limyati DA, Juniar BLL 1998. Jamu Gendong, a kind of tradicional medicine in Indonesia: the microbial contamination of its raw materials and endproduct. J Ethnopharmacol 63: 201-208.

List PH, Schimdt PC 1989. Phytopharmaceutical Technology. Boca Raton:CRC.

Martins HM, Martins ML, Dias MI, Bernardo F 2001. Evaluation of microbiological quality of medicinal plants used in natural infusions. Int J Food Microb 68: 149-151.

Remili H, Boussard P, Devleeschouwer M 1994. Microbiological quality of spray-dried pharmaceutical plant extracts. Eur J Pharm Sci 1: 265-268.

Santos PRV, Oliveira ACX, Tomassini TCB 1995. Controle microbiológico de produtos fitoterápicos. Rev Farm Bioq USP 31: 35-38

Schenkel EP, Simões CMO, Mengue SS, Mentz LA, Irgang BE, Stehmann JR 1985. O espaço das plantas medicinais e suas formas derivadas na medicina científica. Cad Farm 1: 65-72.

Soares LAL 1997. Padronização de extrato aquoso e desenvolvimento de produto seco por aspersão de Phyllantus niruri L. Euphorbiaceae (Quebrapedra) 1997. Dissertação de Mestrado, Curso de Pós-graduação em Ciências Farmacêuticas, Universidade Federal do Rio Grande do Sul.

World Health Organization 1998. Quality control methods for medicinal plant materials. Geneve: WHO. 